\title{
Studies on Intestinal Absorption of Sulpiride (3): Intestinal Absorption of Sulpiride in Rats
}

\author{
Kazuhiro Watanabe,* Tetsuya Sawano, Toshiya JinRIKI, and Juichi Sato \\ Hokkaido College of Pharmacy; 7-1 Katsuraoka-cho, Otaru, Hokkaido 047-0264, Japan. \\ Received July 22, 2003; accepted September 16, 2003
}

\begin{abstract}
The aim of this study was to investigate whether the concomitant administration of the substrates or inhibitors of PEPT1, OCTN1, OCTN2, and P-glycoprotein affects the intestinal absorption of sulpiride in rats. The absorption of sulpiride from rat intestine was decreased by the substrates or inhibitors of PEPT1, OCTN1, and OCTN2. On the other hand, the absorption was increased by the substrates of P-glycoprotein. The effects of these concomitantly administered drugs on the pharmacokinetic behavior of sulpiride after oral administration in rats were investigated. Peak concentration $\left(C_{\max }\right)$ and area under the plasma concentration-time curve $\left(A U C_{0-8 \mathrm{~h}}\right)$ of sulpiride were decreased by the concomitant administration of the substrates or inhibitors of PEPT1, OCTN1, and OCTN2. However, the same parameters were significantly increased by the concomitant administration of the substrates of P-glycoprotein. The present results suggest the possibility of drug-drug interaction during the absorption process in the small intestine due to the coadministration of sulpiride and these agents. These findings provide important information for preventing adverse effects and for ensuring the effectiveness of sulpiride and concomitantly administered drugs.
\end{abstract}

Key words sulpiride; intestine; absorption; transporter; drug-drug interaction

Sulpiride, a dopamine $\mathrm{D}_{2}$ receptor antagonist, is widely used for the treatment of schizophrenia, depression, and gastric and duodenal ulcers because it has a much lower incidence of extrapyramidal side effects than any other antagonist of this type. ${ }^{1,2)}$ Optimization of treatment with sulpiride requires knowledge of its bioavailability, pharmacokinetics, and pharmacodynamics in humans. Mizuno et al. showed that the main site of sulpiride absorption is the intestine. ${ }^{3)}$ The bioavailability of sulpiride shows species differences: it is low in some species, a mean of $27 \%$ in humans and $15-$ $20 \%$ in rat, whereas it is relatively high (44-85\%) in dogs. $\left.{ }^{4}\right)$ The low bioavailability in humans is probably due to poor absorption. ${ }^{3)}$ Baluom et al. ${ }^{5)}$ reported that the intestinal absorption of sulpiride was increased when it was concomitantly administered with the substrates of P-glycoprotein, verapamil, and quinidine in rats. They speculated that the poor oral bioavailability of sulpiride was caused by brush border P-glycoprotein efflux.

In previous studies, we investigated the transcellular transport of sulpiride using the human intestinal cell line Caco-2. ${ }^{6,7)}$ Our results indicated that the apical-to-basolateral transport of sulpiride was significantly inhibited by the substrates or inhibitors of peptide transporter PEPT1, and organic cation transporters OCTN1 and/or OCTN2. On the other hand, the basolateral-to-apical transport was significantly inhibited by the substrates or inhibitors of P-glycoprotein on the apical membrane, and basolateral peptide transporter and organic cation transporter OCT1 on the basolateral membrane. From these findings, we speculated that a decrease in the absorption of sulpiride may occur in combination therapy involving the substrates or inhibitors of PEPT1, OCTN1, and OCTN2; conversely, the concomitant administration of the substrates or inhibitors of P-glycoprotein and OCT1 may increase the absorption of sulpiride.

To determine the appropriate clinical administration of sulpiride, we consider it essential to investigate its interactions with drug transporters. Drug transporters corresponding to human drug transporters such as P-glycoprotein, or- ganic cation and anion transporters, and peptide transporters are expressed in the rat intestine. The aim of this study was to investigate whether the concomitant administration of sulpiride and the substrates or inhibitors of PEPT1, OCTN1, OCTN2, OCT1, and P-glycoprotein affects the intestinal absorption and the pharmacokinetic behavior of sulpiride after oral administration in rats.

\section{MATERIALS AND METHODS}

Materials Sulpiride and cephalexin hydrate were purchased from ICN Biomedicals, Inc. (Costa Mesa, CA, U.S.A.). L-Carnitine was purchased from Sigma (St. Louis, MO, U.S.A.). Captopril, carboxymethyl cellulose sodium salt (CMC-Na), choline chloride, cyclosporin A, quinidine, verapamil hydrochloride, and nicotinic acid were purchased from Wako Pure Chemical Industries (Osaka, Japan). Guanidine was purchased from Kanto Chemical (Tokyo, Japan). All chemicals used were of the highest purity commercially available.

Conditions for High-Performance Liquid Chromatography (HPLC) HPLC $^{6}$ was carried out using a model CCPM pump (Tosoh, Tokyo, Japan) equipped with an RF530 fluorescence detector (Shimadzu, Kyoto, Japan). The excitation and emission wavelengths were set at 307 and $358 \mathrm{~nm}$, respectively. A reverse-phase column was used at $50{ }^{\circ} \mathrm{C}$, and the mobile phase consisted of a mixture of acetonitrile/1\% triethanolamine/ $6 \mathrm{mM} \mathrm{H}_{3} \mathrm{PO}_{4}(3 / 0.3 / 3, \mathrm{v} / \mathrm{v} / \mathrm{v})$. Calibration curves were obtained by injecting sulpiride (2$4000 \mathrm{ng}$ ) containing a quantitative amount (300 ng) of 1naphthylamine as an internal standard into the HPLC system.

Animals Animal studies were performed in accordance with "The Guidelines for the Care and Use of Laboratory Animals, Hokkaido College of Pharmacy." All animals were housed in individual cages in a room with controlled temperature $\left(23 \pm 1^{\circ} \mathrm{C}\right)$, humidity $(55 \pm 5 \%)$ and light $(06: 00$ 18:00 h), and were maintained on an MF laboratory diet (Oriental Yeast, Tokyo, Japan) and water ad libitum. Male Wistar 
rats (9-10 weeks old) were purchased from Japan SLC (Shizuoka, Japan).

Absorption Studies. 1) Intestinal Administration: Male Wistar rats were used after they were fasted for $20 \mathrm{~h}$. Intestinal absorption studies were carried out using the in situ loop (3 loops, each $10 \mathrm{~cm}$ ) method of Saitoh et al. ${ }^{8}$ Sulpiride and other compounds were suspended in $0.1 \mathrm{M}$ phosphate buffer ( $\mathrm{pH}$ 7.0). After gently washing out the contents of the loop with $20 \mathrm{ml}$ of saline, sulpiride suspension with or without other compounds was injected into each loop with a syringe. After $1 \mathrm{~h}$, the loop was cut off under ether anesthesia and rinsed with ice-cold saline. The mucosa of each loop was scraped and homogenized with saline. After the addition of 1-nahpthylamine $(100 \mu \mathrm{g} / 0.1 \mathrm{ml})$ as an internal standard, the contents and the mucosa of the loop were emptied into a 10$\mathrm{ml}$ volumetric flask. Three milliliters of $0.2 \mathrm{M} \mathrm{NaHCO}_{3} /$ $\mathrm{Na}_{2} \mathrm{CO}_{3}$ buffer ( $\mathrm{pH} 9.8$ ) and $5 \mathrm{ml}$ of chloroform were added to $2 \mathrm{ml}$ of the sample. After mixing for $10 \mathrm{~min}$ in a reciprocal shaker, the mixture was centrifuged at $5000 \mathrm{rpm}$ for $10 \mathrm{~min}$, and $4 \mathrm{ml}$ of the chloroform phase was evaporated to dryness using a rotary evaporator. The residue was dissolved in $0.3 \mathrm{ml}$ of methanol and filtered through a Millipore filter (Millex-LG, Millipore, U.S.A.). An aliquot of the filtrate was injected into the HPLC system. To confirm the validity of the in situ loop method, known amounts of sulpiride were added to duodenum, jejunum, and ileum loops. The recoveries of sulpiride from each loop were approximately $95 \%$.

2) Oral Administration: In experiments involving oral administration, sulpiride was administered at a dose of $100 \mathrm{mg} / \mathrm{kg}$ to rats. Sulpiride and other compounds were suspended in $0.5 \%$ CMC-Na. Blood samples $(0.15 \mathrm{ml})$ were collected from the jugular vein under light ether anesthesia. The samples were immediately centrifuged for $15 \mathrm{~min}$ at $6000 \mathrm{rpm}$ to obtain plasma $(0.05 \mathrm{ml})$, which was kept frozen at $-20^{\circ} \mathrm{C}$ until analysis. To $0.05 \mathrm{ml}$ of plasma samples were added $0.35 \mathrm{ml}$ of $0.2 \mathrm{M} \mathrm{NaHCO} / \mathrm{Na}_{2} \mathrm{CO}_{3}$ buffer ( $\mathrm{pH} 9.8$ ), $0.6 \mathrm{ml}$ of chloroform, and $0.1 \mu \mathrm{g}$ of 1-naphthylamine as an internal standard. After mixing for $10 \mathrm{~min}$ in a reciprocal shaker, the mixture was centrifuged at $5000 \mathrm{rpm}$ for $10 \mathrm{~min}$, and $0.5 \mathrm{ml}$ of the chloroform phase was evaporated to dryness using a rotary evaporator. The residue was dissolved in $0.05 \mathrm{ml}$ of the mobile phase and filtered through a Millipore filter (Millex-LG). An aliquot of the filtrate was injected into the HPLC system. Known amounts of sulpiride were added to male Wistar rat plasma, and sulpiride recovered by the liquid-liquid extraction method was measured. The recoveries of sulpiride from the plasma were approximately $95 \%$.

Data Analysis The results are expressed as the mean \pm S.D. Differences between two groups were evaluated using Student's $t$-test or Welch's $t$-test after the initial $F$-test analysis. Three or more groups were evaluated using Bonferroni/Dunn's multiple-comparison test or Scheffe's multiplecomparison test after the Bartlett test and the one-way analysis of variance. $p$ values less than 0.05 were considered significant.

Peak concentration $\left(C_{\max }\right)$ was determined from the plasma profile. The area under the plasma concentration-time curve from 0 to $8 \mathrm{~h}\left(A U C_{0-8 \mathrm{~h}}\right)$ was calculated according to the trapezoidal rule. The mean residence time (MRT) was calculated based on the statistical moment theory. ${ }^{9)}$

\section{RESULTS AND DISCUSSION}

In Situ Absorption Study We investigated the absorption of sulpiride from duodenum, jejunum, and ileum loops and the effects of substrates or inhibitors of various drug transporters on the absorption from each loop in rats. Sulpiride $(0.3 \mathrm{mg} / 0.5 \mathrm{ml})$ was injected in each loop of rat small intestine. The disappearance rate (\%) of sulpiride was calculated from the amount of sulpiride remaining in each loop after $1 \mathrm{~h}$. As shown in Table 1, the disappearance rate of sulpiride was the highest in the duodenum at approximately $32 \%$, followed by those in the jejunum and ileum at approximately $20 \%$ and $10 \%$, respectively. This suggests that the main site of the intestinal absorption of sulpiride in rats is the duodenum, similar to the case in rabbits. ${ }^{10)}$ The disappear-

Table 1. Disappearance Rates of Sulpiride from the Duodenum, Jejunum, and Ileum of Male Wistar Rats

\begin{tabular}{|c|c|c|c|c|}
\hline \multirow{2}{*}{ Dose } & \multirow{2}{*}{$\begin{array}{l}\text { Drug } \\
\text { (mg) }\end{array}$} & \multicolumn{3}{|c|}{ Disappearance $\left.(\% \text { of dose })^{a}\right)$} \\
\hline & & Duodenum & Jejunum & Ileum \\
\hline Control & & $32.0 \pm 4.1$ & $20.2 \pm 4.0$ & $10.1 \pm 3.2$ \\
\hline \multirow[t]{2}{*}{ Captopril } & 0.3 & $17.5 \pm 3.2 * *$ & $11.9 \pm 1.0 *$ & $7.9 \pm 2.0$ \\
\hline & 1.0 & $7.0 \pm 2.1 * * *$ & $10.8 \pm 1.4 * *$ & $2.1 \pm 1.1 * *$ \\
\hline \multirow[t]{2}{*}{ Cephalexin } & 0.3 & $13.2 \pm 3.0^{* * *}$ & $14.9 \pm 6.9$ & $6.8 \pm 1.9$ \\
\hline & 1.0 & $11.7 \pm 1.8^{* * *}$ & $7.2 \pm 3.3 *$ & $2.4 \pm 1.2 * *$ \\
\hline \multirow[t]{2}{*}{ Choline } & 0.3 & $30.4 \pm 3.0$ & $24.1 \pm 1.5$ & $15.1 \pm 3.7$ \\
\hline & 1.0 & $23.0 \pm 3.6^{*}$ & $24.4 \pm 4.4$ & $13.5 \pm 1.6$ \\
\hline \multirow[t]{2}{*}{ L-Carnitine } & 0.3 & $22.2 \pm 2.1 *$ & $13.4 \pm 2.6$ & $8.7 \pm 0.9$ \\
\hline & 1.0 & $19.8 \pm 2.9 * *$ & $12.9 \pm 2.9$ & $8.1 \pm 2.5$ \\
\hline \multirow[t]{2}{*}{ Guanidine } & 0.3 & $26.7 \pm 3.4$ & $16.7 \pm 1.3$ & $10.7 \pm 3.6$ \\
\hline & 1.0 & $22.2 \pm 6.1$ & $22.1 \pm 2.6$ & $9.3 \pm 4.4$ \\
\hline \multirow[t]{2}{*}{ Nicotinic acid } & 0.3 & $29.5 \pm 4.3$ & $20.0 \pm 8.2$ & $14.4 \pm 10.5$ \\
\hline & 1.0 & $21.5 \pm 6.5$ & $16.9 \pm 2.2$ & $8.4 \pm 2.7$ \\
\hline \multirow[t]{2}{*}{ Cyclosporin A } & 0.3 & $40.5 \pm 3.2 *$ & $35.7 \pm 3.1 * * *$ & $27.2 \pm 5.4 * * *$ \\
\hline & 1.0 & $46.1 \pm 2.6^{* *}$ & $42.3 \pm 1.0 * * *$ & $34.6 \pm 1.5 * * *$ \\
\hline \multirow[t]{2}{*}{ Quinidine } & 0.3 & $43.5 \pm 6.5^{*}$ & $39.3 \pm 4.0 *$ & $30.8 \pm 2.3 * * *$ \\
\hline & 1.0 & $52.9 \pm 4.6^{* *}$ & $50.5 \pm 11.0 * * *$ & $37.1 \pm 3.4 * * *$ \\
\hline \multirow[t]{2}{*}{ Verapamil } & 0.3 & $41.3 \pm 6.2$ & $25.7 \pm 2.5$ & $16.0 \pm 4.9$ \\
\hline & 1.0 & $50.5 \pm 3.2 * *$ & $36.4 \pm 3.0 * * *$ & $27.1 \pm 6.3 * *$ \\
\hline
\end{tabular}

Sulpiride $(0.3 \mathrm{mg} / 0.5 \mathrm{ml})$ was injected with or without various drugs into duodenum, jejunum, and ileum loops in rats. After $1 \mathrm{~h}$, sulpiride in loop content and mucosa was determined using HPLC. The value obtained by subtracting the total amount of sulpiride in loop content and mucosa from the injected dose was assumed to be the disappearance. a) Disappearance $(\%$ of dose $)=\{$ injected dose $-($ loop content + mucosa $)\}$ injected dose $\times 100$. Each value represents the mean \pm S.D. for $3-5$ loops. Significantly different from the control, $* p<0.05, * * p<0.01, * * * p<0.001$.

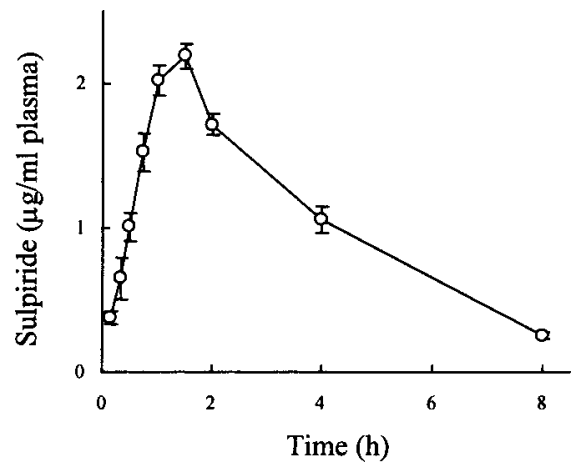

Fig. 1. Plasma Concentration-Time Curve of Sulpiride after Oral Administration to Male Wistar Rats

Sulpiride was administered at a dose of $100 \mathrm{mg} / \mathrm{kg}$. $C_{\max }$ was $2.16 \pm 0.09 \mathrm{mg} / \mathrm{ml}$, $A U C_{0-8 \mathrm{~h}}$ was $8.25 \pm 0.35 \mathrm{mg} / \mathrm{ml} \cdot \mathrm{h}$, and MRT was $2.83 \pm 0.05 \mathrm{~h}$. Each value represents the mean \pm S.D. of 14 rats. 
ance of sulpiride from duodenum and ileum loops was significantly and markedly decreased by captopri1 ${ }^{11)}$ and cephalexin, ${ }^{12)}$ substrates of PEPT1, respectively. The disappearance rate from duodenum loops was significantly decreased by choline ${ }^{13,14)}$ (only $1.0 \mathrm{mg}$ ), the substrate and inhibitor of OCT1, OCTN1, and OCTN2, and L-carnitine, ${ }^{13)}$ the substrate and inhibitor of OCTN1 and OCTN2. Conversely, the disappearance rate from duodenum, jejunum, and ileum loops was significantly increased by cyclosporin $\mathrm{A},{ }^{15}$ quinidine and verapamil, ${ }^{14,16-18)}$ the substrate of P-glycoprotein. Quinidine and verapamil ${ }^{14,16-18)}$ are also substrates of the uptake transporters OCTN1 and OCTN2. In the present study, however, both compounds increased the disappearance rate of sulpiride from the intestinal loops. The results suggest that quinidine and verapamil inhibit the efflux transporter Pglycoprotein more than the uptake transporters OCTN1 and OCTN2. However, guanidine, ${ }^{19)}$ the substrate of OCT3, and nicotinic acid ${ }^{20)}$ the substrate of AE2 and MCT1 did not affect the absorption of sulpiride.

Plasma Sulpiride Concentration after Oral Administration Figure 1 shows the plasma concentration-time curve of sulpiride after administration to rats at a dose of $100 \mathrm{mg} / \mathrm{kg}$. The peak concentration $\left(C_{\max }\right)$, area under the plasma concentration-time curve from 0 to $8 \mathrm{~h}\left(A U C_{0-8 \mathrm{~h}}\right)$, and MRT of sulpiride are listed in Table 2. It has been reported that there are some species differences in the bioavailability of sulpiride: the bioavailability of sulpiride in rats and humans is approximately $15 \%{ }^{3,4)}$ and $27 \%,{ }^{4)}$ respectively,
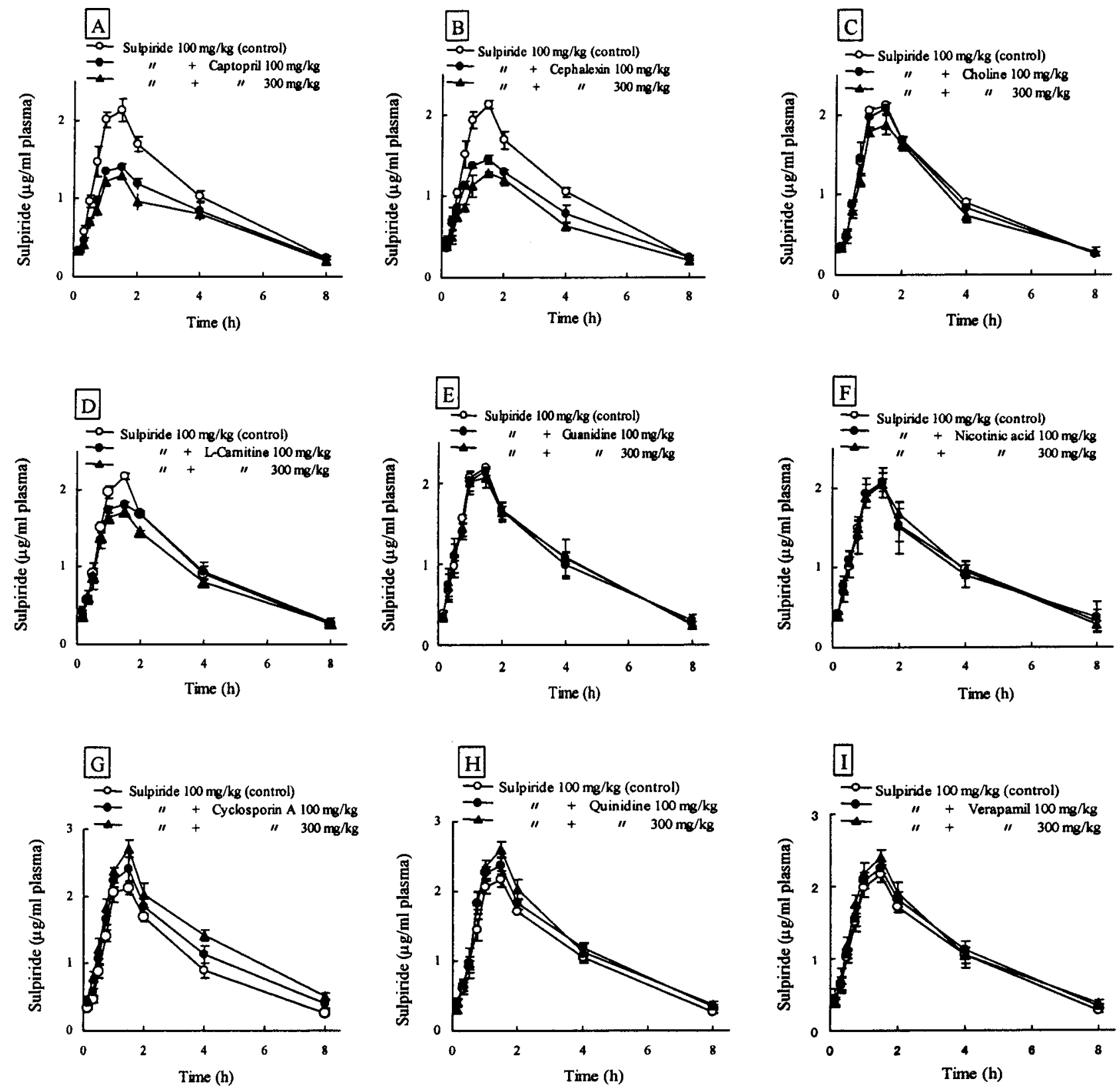

Fig. 2. Plasma Concentration-Time Curves of Sulpiride after Oral Administration to Male Wistar Rats with or without Substrates or Inhibitors of Various Drug Transporters

Sulpiride (100 mg/kg) was administered with or without captopril (A), cephalexin (B), choline (C), L-carnitine (D), guanidine (E), nicotinic acid (F), cyclosporin A (G), quinidine $(\mathrm{H})$, and verapamil (I) at a dose of 100 or $300 \mathrm{mg} / \mathrm{kg}$. Each value represents the mean \pm S.D. of $3-4$ rats. 
whereas that in dogs is $44-68 \%$ and $75-85 \%$ and is thus higher than those in rats and humans. ${ }^{4}$ The difference in bioavailability with dosage form is also notable; for example, the bioavailability in rabbits is 18,25 , and $46 \%$ in liquid form, powder form, and tablet form, respectively. ${ }^{10)}$ Furthermore, it is known that the bioavailability differs depending on the solvent used for suspension or dissolution. CMC-Na, $0.5 \%$ acetate $(\mathrm{pH} 4.5),{ }^{3)}$ and $0.1 \mathrm{~m}$ phosphate buffer $(\mathrm{pH}$ $7.0)^{10)}$ are used as suspending and dissolving agents for sulpiride. When sulpiride suspension in $0.2 \% \mathrm{CMC}-\mathrm{Na}$ was orally administered to rats, intestinal absorption was very low and bioavailability was approximately 3\%; however, when sulpiride was dissolved in $0.5 \%$ acetic acid solution (pH 4.5), bioavailability was $15 \%{ }^{3)}$ In the present study, we prepared impalpable powdered sulpiride with an agate mortar and pestle and then orally administered it to rats as sulpiride suspension in $0.5 \%$ CMC-Na. $C_{\max }$ and $A U C_{0-8 \mathrm{~h}}$ were slightly lower than those obtained ${ }^{3)}$ when sulpiride was dissolved in $0.5 \%$ acetic acid solution.

The effects of coadministration of the substrates or inhibitors of various drug transporters on plasma sulpiride concentration after oral administration were investigated and the pharmacokinetic parameters were compared. The plasma concentration of sulpiride was markedly decreased compared with the control groups when it was concomitantly administered with captopril and cephalexin (Figs. 2A, B), and the plasma concentration was also slightly decreased compared with the control groups when it was concomitantly administered with choline $(300 \mathrm{mg} / \mathrm{kg})$ and L-carnitine (Figs. 2C, D). The calculated pharmacokinetic parameters are listed in Table 2. $C_{\max }$ and $A U C_{0-8 \mathrm{~h}}$ for sulpiride in the captopril and cephalexin coadministration groups were significantly and markedly reduced, and those in the choline and L-carnitine (both $300 \mathrm{mg} / \mathrm{kg}$ ) coadministration groups were significantly and dose dependently reduced compared with the control groups. In contrast, guanidine and nicotinic acid did not have any significant effect on the plasma concentration and the pharmacokinetic parameters of sulpiride (Figs. 2E, F and Table 2). On the other hand, the plasma concentrations of sulpiride after concomitant administration of cyclosporin A and quinidine were increased, and the plasma concentration after concomitant administration of verapamil was slightly increased compared with the control groups (Figs. 2G, H, I). $C_{\max }$ and $A U C_{0-8 \mathrm{~h}}$ for sulpiride in the cyclosporin $\mathrm{A}$ or quinidine coadministration groups were significantly increased compared with the control groups, and $A U C_{0-8 \mathrm{~h}}$ for sulpiride in the verapamil $(300 \mathrm{mg} / \mathrm{kg})$ coadministration group was significantly increased compared with the control group. No compounds affected MRT. The results support those indicating the disappearance of sulpiride from the intestinal loops in the in situ loop method. Recently, Baluom et $a .^{5)}$ reported that the bioavailability of sulpiride was increased by the concomitant administration of quinidine and verapamil in rats. They indicated that the increase depended on the inhibition of P-glycoprotein-transported sulpiride secretion by quinidine and verapamil. Part of our results in the present study agreed with those of Baluom et al. ${ }^{5}$ )

The disappearance rates of sulpiride from intestinal loops and plasma sulpiride concentration after oral administration were decreased by the concomitant administration of captopril and cephalexin, the substrate of PEPT1, and L-carnitine,
Table 2. Pharmacokinetic Parameters of Sulpiride after Oral Administration to Male Wistar Rats with or without Substrates or Inhibitors of Various Drug Transporters

\begin{tabular}{|c|c|c|c|c|}
\hline Drug & $\begin{array}{c}\text { Dose } \\
(\mathrm{mg} / \mathrm{kg})\end{array}$ & $\begin{array}{c}C_{\max } \\
(\mu \mathrm{g} / \mathrm{ml})\end{array}$ & $\begin{array}{l}A U C_{0-8 \mathrm{~h}} \\
(\mu \mathrm{g} / \mathrm{ml} \cdot \mathrm{h})\end{array}$ & $\begin{array}{l}\text { MRT } \\
\text { (h) }\end{array}$ \\
\hline \multirow[t]{3}{*}{ Captopril } & - & $2.13 \pm 0.14$ & $8.20 \pm 0.95$ & $2.82 \pm 0.04$ \\
\hline & 100 & $1.39 \pm 0.10 * * *$ & $6.15 \pm 0.21 * *$ & $2.99 \pm 0.24$ \\
\hline & 300 & $1.33 \pm 0.06 * * *$ & $5.68 \pm 0.49 * *$ & $3.06 \pm 0.06$ \\
\hline \multirow[t]{3}{*}{ Cephalexin } & - & $2.13 \pm 0.05$ & $8.31 \pm 0.70$ & $2.83 \pm 0.14$ \\
\hline & 100 & $1.46 \pm 0.11 * * *$ & $6.34 \pm 0.35^{*}$ & $2.91 \pm 0.05$ \\
\hline & 300 & $1.27 \pm 0.07 * * *$ & $5.34 \pm 0.52 * *$ & $2.87 \pm 0.11$ \\
\hline \multirow[t]{3}{*}{ Choline } & - & $2.12 \pm 0.10$ & $7.93 \pm 0.40$ & $3.14 \pm 0.71$ \\
\hline & 100 & $2.10 \pm 0.05$ & $7.55 \pm 0.29$ & $3.00 \pm 0.37$ \\
\hline & 300 & $1.85 \pm 0.10 *$ & $7.00 \pm 0.05^{*}$ & $2.82 \pm 0.02$ \\
\hline \multirow[t]{3}{*}{ L-Carnitine } & - & $2.17 \pm 0.04$ & $7.93 \pm 0.78$ & $2.80 \pm 0.03$ \\
\hline & 100 & $1.82 \pm 0.10 * *$ & $7.74 \pm 0.28$ & $2.88 \pm 0.12$ \\
\hline & 300 & $1.72 \pm 0.09 * *$ & $6.52 \pm 0.36^{*}$ & $2.82 \pm 0.14$ \\
\hline \multirow[t]{3}{*}{ Guanidine } & - & $2.16 \pm 0.06$ & $8.38 \pm 0.51$ & $2.84 \pm 0.04$ \\
\hline & 100 & $2.15 \pm 0.09$ & $8.23 \pm 0.47$ & $2.82 \pm 0.25$ \\
\hline & 300 & $2.10 \pm 0.09$ & $8.17 \pm 0.67$ & $2.83 \pm 0.25$ \\
\hline \multirow[t]{3}{*}{ Nicotinic acid } & - & $2.15 \pm 0.06$ & $8.20 \pm 0.25$ & $2.82 \pm 0.11$ \\
\hline & 100 & $2.13 \pm 0.17$ & $8.26 \pm 0.33$ & $2.81 \pm 0.06$ \\
\hline & 300 & $2.05 \pm 0.08$ & $8.12 \pm 0.76$ & $2.79 \pm 0.07$ \\
\hline \multirow[t]{3}{*}{ Cyclosporin A } & A - & $2.12 \pm 0.10$ & $7.93 \pm 0.78$ & $3.14 \pm 0.71$ \\
\hline & 100 & $2.45 \pm 0.11 *$ & $9.37 \pm 0.77 *$ & $2.96 \pm 0.08$ \\
\hline & 300 & $2.71 \pm 0.12 * *$ & $10.90 \pm 0.56 * *$ & $3.07 \pm 0.03$ \\
\hline \multirow[t]{3}{*}{ Quinidine } & - & $2.17 \pm 0.09$ & $8.48 \pm 0.58$ & $2.85 \pm 0.05$ \\
\hline & 100 & $2.44 \pm 0.07 *$ & $9.39 \pm 0.55$ & $2.91 \pm 0.07$ \\
\hline & 300 & $2.60 \pm 0.12 * *$ & $10.36 \pm 0.63 *$ & $2.88 \pm 0.07$ \\
\hline \multirow[t]{3}{*}{ Verapamil } & - & $2.18 \pm 0.07$ & $8.36 \pm 0.55$ & $2.83 \pm 0.06$ \\
\hline & 100 & $2.29 \pm 0.14$ & $8.99 \pm 0.45$ & $2.91 \pm 0.06$ \\
\hline & 300 & $2.40 \pm 0.11$ & $9.73 \pm 0.78 *$ & $2.89 \pm 0.12$ \\
\hline
\end{tabular}

Sulpiride $(100 \mathrm{mg} / \mathrm{kg})$ was administered with or without captopril, cephalexin, choline, L-carnitine, guanidine, nicotinic acid, cyclosporin A, quinidine, and verapamil at a dose of 100 or $300 \mathrm{mg} / \mathrm{kg}$. Each value represents the mean \pm S.D. for $3-4$ rats. Significantly different from the control, $* p<0.05, * * p<0.01$, *** $p<0.001$.

the substrate of OCTN1 and OCTN2, and increased by the concomitant administration of cyclosporin A, the substrate of P-glycoprotein. In conclusion, the present results suggest the possibility of drug-drug interaction between sulpiride and these substrates of drug transporters; it is considered that the plasma sulpiride concentration after oral administration is decreased by the concomitant administration of the substrates of PEPT1, OCTN1, and OCTN2, and conversely increased by the concomitant administration of the substrate of P-glycoprotein. These findings provide important information for preventing adverse effects and for ensuring the effectiveness of sulpiride and concomitantly administered drugs.

\section{REFERENCES}

1) Jenner P., Marsden C. D., Neuropharmacology, 20, 1285-1293 (1981).

2) Trabucchi M., Longoni R., Fresia P., Spano P. F., Life Sci., 17, 15511556 (1975).

3) Mizuno N., Morita E., Nishikata M., Shinkuma D., Yamanaka Y., Arch. Int. Pharmacodyn. Ther, 283, 30-38 (1986).

4) Bateman D. N., Adv. Biochem. Psychopharmacol., 35, 143-162 (1982).

5) Baluom M., Friedman M., Rubinstein A., J. Control. Rel., 70, 139147 (2001).

6) Watanabe K., Sawano T., Terada K., Endo T., Sakata M., Sato J., Biol. Pharm. Bull., 25, 885-890 (2002).

7) Watanabe K., Sawano T., Endo T., Sakata M., Sato J., Biol. Pharm. Bull., 25, 1345-1350 (2002).

8) Saitoh H., Hatakeyama M., Eguchi O., Oda M., Takada M., J. Pharma- 
col. Sci., 87, 73-75 (1998).

9) Yamaoka K., Nakagawa T., Uno T., J. Pharmacokinet. Biopharm., 6, 547-558 (1978).

10) Kohri N., Naasani I., Iseki K., Miyazaki K., J. Pharm. Pharmacol., 48, 371-374 (1996).

11) Boll M., Markovich D., Weber W. M., Korte H., Daniel H., Murer H., Pflugers Arch., 429, 146-149 (1994).

12) Terada T., Saito H., Inui K., J. Biol. Chem., 273, 5582-5585 (1998).

13) Endou H., Folia Pharmacol. Jpn., 116, 114-124 (2000).

14) Yabuuchi H., Tamai I., Nezu J., Sakamoto K., Oku A., Shimane M., Sai Y., Tsuji A., J. Pharmacol. Exp. Ther., 289, 768-773 (1999).
15) Saeki T., Ueda K., Tanagawara Y., Hori R., Komano T., J. Biol. Chem., 268, 6077-6080 (1993).

16) Wandel C., Kim R. B., Kajiji S., Guengerich F. P., Wilkinson G. R., Wood A. J., Cancer Res., 59, 3944-3948 (1999).

17) Hedman A., Meijer D. K. F., J. Pharm. Sci., 87, 457-461 (1998).

18) Shu Y., Bello C. L., Mangravite L. M., Feng B., Giacomini K. M., J. Pharmacol. Exp. Ther., 299, 392-398 (2001).

19) Kekuda R., Prasad P. D., Wu P., Wang H., Fei Y. J., Leibach F. H., Ganapathy V., J. Biol. Chem., 273, 15971-15979 (1998).

20) Tamai I., Yakugaku Zasshi, 117, 415-434 (1997). 